EDITORIAL

For reprint orders, please contact: reprints@futuremedicine.com

\section{Can we predict and prevent specific sites of metastases in breast cancer patients?}
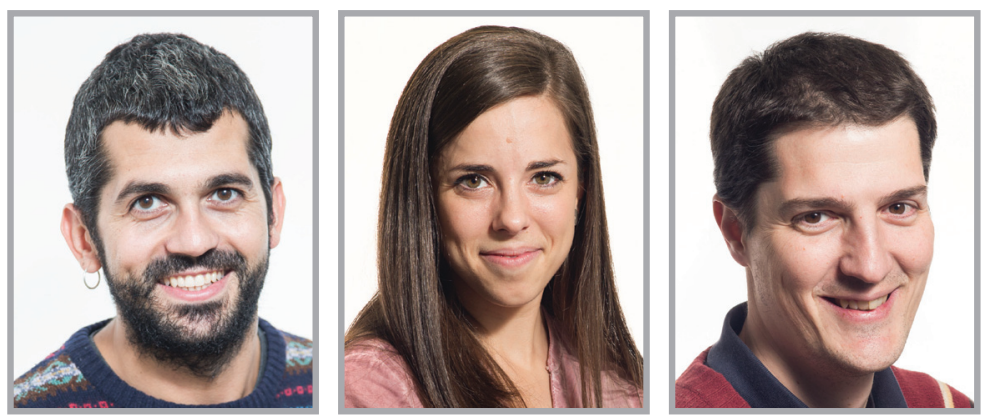

Fernando Salvador ${ }^{\ddagger, 1}$, Anna Bellmunt ${ }^{\ddagger 1}$ \& Roger R Gomis ${ }^{*, 1,2}$

First draft submitted: 29 January 2016; Accepted for publication: 3 February 2016; Published online: 18 April 2016

Despite improvements in breast cancer therapies, cancer cells frequently spread to distant organs years or decades after primary tumor surgery and adjuvant treatment. This expansion, known as metastasis, can bring about fatal consequences. Traditionally, the risk of metastasis has been predicted by prognostic factors such as tumor size, axillary lymph node status and histological grade. More recently, genomic tests have also been used for this purpose. The presence of ER, PR and $E R B B 2$ gene amplification are currently key markers in the characterization of breast tumor type that drive the selection of specific therapies [1]. ER-positive tumors are more prone to metastasize into the bone, whereas ER-negative tumors preferentially spread to visceral organs such as lung, liver and brain [2]. However, the reliability of these markers is limited. In this regard, substantial efforts have been made to find new markers that predict the most probable target organ of metastasis, with the aim to improve diagnosis and develop organ-specific treatments for breast cancer metastatic patients.

Metastasis is an inefficient process through which cancer cells must overcome several hurdles to establish a secondary lesion in a distant site. These steps involve intravasation into the blood stream, extravasation into a distant tissue and colonization of the target organ. Colonization involves cancer cell-host tissue interactions, evasion of the immune system, activation of cytokine signaling and extracellular matrix modifications that allow tumor cells to complete metastatic growth. This set of activities supports the establishment of a new metastasis that mimics the formation of a new and independent tumor entity [3]. Interestingly, recent evidence supports the notion that modifications of the microenvironment of distant organs are required prior to the tumor cells reaching the metastatic site. The preparation of a 'premetastatic niche' suitable for the reception and growth of metastatic cells may be needed [4]. These

\title{
Breast Cancer Management
}

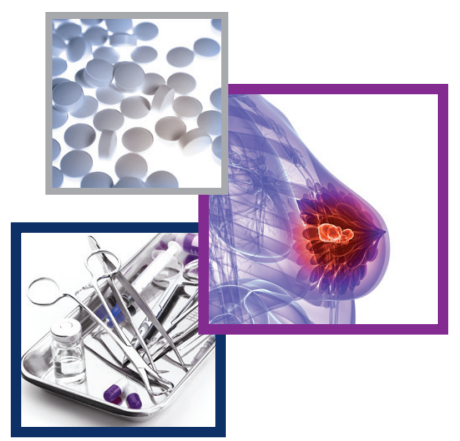

\section{KEYWORDS}

- breast cancer • organ-specific metastasis $\bullet$ predictive markers

“...substantial efforts have been made to find new markers that predict the most probable target organ of metastasis, with the aim to improve diagnosis and develop organ-specific treatments for breast cancer metastatic patients."

'Oncology Program, Institute for Research in Biomedicine (IRB Barcelona), The Barcelona Institute of Science 


\section{“The metastasis gene signatures from primary tumors identified in the last decade provide relevant information about the mechanisms underlying metastasis mechanisms, and tissue specificity."}

lines of evidence collectively validate the initial 'seed and soil' hypothesis promulgated by Steven Paget in the 19th century, suggesting that the local microenvironment of a specific tissue is more accessible, fitted and hence permissive than others for the establishment and colonization of a given tumor cell [5].

During the past 15 years, several studies have identified sets of genes whose expression is associated with metastasis - some in a tissue-specific manner. Unfortunately, most of these genes have failed to provide new diagnostic tools to stratify patients on the basis of risk of distant relapse and tissue-specific metastasis. The absence of primary tumor sample cohorts for which clinical annotations of site-specific time to metastasis are available, as well as feasibility issues regarding the collection of metastatic biopsies, has become a major limitation. These limitations, in turn, are magnified when the prognostic/predictive power of genes associated with metastasis is restricted to the primary tumor. Many tissuespecific metastasis genes may be gained or lost at the distant site where tissue-specific functions are need. Contrary, genes whose expression changes at the primary site and that are associated with metastasis may confer both a specific advantage for growth at the primary site and beyond once disseminated to specific sites. Alternatively, these genes can be ascribed to clones within the heterogeneity of primary tumor populations [6]. These clones have site-specific advantages with respect to settling at the distant site that they have migrated to and where they are expanded and dominant.

Several genes contribute to the lung metastasis signature in primary tumors [7]. Elevated expression of EREG, PTGS2, MMP1 and cytokine ANGPTL4 increases breast cancer cell extravasation in lung capillaries [8,9]. Lung-tropic breast cancer cells also express VCAM, which interacts with macrophages and enhances cell survival by activating PI3K-AKT signaling [10]. Similarly, the downregulation of RARRES3 facilitates the adhesion of breast cancer cells to the extracellular matrix proteins of lung parenchyma and suppresses tumor cell differentiation, thereby favoring metastasis initiation [11]. All these genes, beyond their predictive value in the clinical setting, are potential candidates for the prevention and therapeutic intervention of metastasis. But it is unclear when, where and how they could be effective.

A large subset of breast cancer patients suffers from bone metastasis as the first site of relapse, and for these individuals the disease is largely confined to bone during the course of the disease [12]. Due to the fenestrated endothelia, bone marrow sinusoids are more permissive to the homing of tumor cells than the capillaries of other tissues. In the bone, breast cancer cells can take advantage of several factors secreted by bone matrix cells, such as chemokine CXCL12, to activate a survival-signaling pathway. In fact, tumor cells with elevated SRC signaling activity and high levels of CXCR4, a receptor for CXCL12, are preferentially favored by survival signals in the bone marrow, and the expression of this receptor is associated with breast cancer bone relapse [13-15]. To generate an overt metastasis, a variety of factors such as PTHRP, TNF- $\alpha$ and IL-6/11 are secreted by tumor cells, stimulating the production of RANKL from osteoblasts. RANKL activates its receptor RANK to promote osteoclast differentiation. Osteoclast activation is a hallmark of osteolysis development and promotes bone resorption. The osteolytic process causes the release of bone matrix growth factors into the microenvironment (i.e., TGF- $\beta$ ), thus stimulating tumor cell growth. This 'vicious cycle' gives rise to aggressive tumor cells in bone metastasis $[16,17]$. The expression of these factors correlates with poor prognosis and bone relapse in some breast cancer patients $[18,19]$; however, it fails to predict the risk of bone metastasis in early stage tumors [20].

The identification of new predictive molecular biomarkers in nonadvanced tumors is of emerging clinical interest. Bisphosphonates and Denosumab have proven effective in the management of the morbidity of skeletal-related events morbidity. However, these treatments do not improve disease progression or overall survival rates [21]. Interestingly, recent evidence showed that 16q23 genomic gain in early stage primary tumors is associated with a high risk of developing bone metastasis and with poor overall survival. The $M A F$ gene was identified as the genetic driver of the 16q23 region. In fact, breast cancer patients with high MAF expression (mRNA and protein) have a higher cumulative risk of metastasis to bone but not to other organs. Moreover, functional validation and mechanistic studies showed that MAF acts as a transcription factor to control the expression of a gene program, including functions such as migration, adhesion and tumor cell-stroma interaction. Among these genes, PTHrP was identified as an important element 
for MAF-driven bone metastasis [22]. This novel finding opens up new therapeutic strategies in breast cancer. Bone microenvironment modifying agents such as biphosphonates and the antiRANK ligand antibody Denosumab have the theoretical potential to prevent bone metastasis, albeit data from clinical trials are as yet inconclusive in unselected patient populations [21,23]. The identification of a biomarker that predicts bonespecific metastasis in breast tumors in a timely manner has raised the possibility of including such agents in the adjuvant setting to effectively prevent dissemination and bone metastases in MAF-expressing breast cancer patients [22].

While breast cancer metastasis to the liver and brain is less frequent than to the bone, the former have the worst outcome. Similarly to the bone, the hepatic endothelium is permissive for cancer cell extravasation. In this process, adhesion molecules are involved in the establishment of metastasis. Claudin-2 plays a key role in mediating the interaction between hepatocytes and cancer cells promoting the activation of metastatic signaling pathways. Indeed, Claudin-2 expression is considered a poor prognosis factor that mediates breast cancer relapse to the liver [24,25].

In contrast to bone and liver, the brain is the most difficult organ to access by breast cancer cells due to the presence of the blood-brain barrier (BBB). Consequently, most brain metastasis mediators are adhesion-, extravasation- and survival-related genes [26,27]. On the other hand, the presence of the $\mathrm{BBB}$ also limits drug delivery, thus impeding effective brain metastasis treatment. Recently, it has been suggested that patients with the HER2-enriched breast cancer subtype treated with Trastuzumab develop a higher risk of metastasis to the brain compared with other organs. New small drugs that penetrate the BBB, including lapatinib, are being used in the advance setting treatment [28].
Recent years have witnessed a significant improvement in breast cancer therapy directed at reducing primary tumor growth; however, distant metastasis has emerged as a new problem. Current therapies, mainly aimed at the primary tumor, are not as effective at preventing and controlling metastasis to distant organs. The metastasis gene signatures from primary tumors identified in the last decade provide relevant information about the mechanisms underlying metastasis mechanisms, and tissue specificity. This information may eventually allow the identification of patients who can benefit from the inclusion of therapies seeking to prevent tissue-specific metastasis. The integration of these predictive markers in routine clinical practice opens up new avenues in an era of personalized medicine. In addition, the development of organ-specific metastatic animal models would contribute to establishing preclinical systems to functionally validate metastasis biomarkers, thus providing an invaluable tool with which to study organ-specific metastasis and to develop new therapies.

Financial \& competing interests disclosure

A Bellmunt is supported by an FPI-Severo Ochoa fellowship and F Salvador is supported by a Juan de la Cierva research contract by the Spanish Government. RR Gomis is supported by the Institució Catalana de Recerca $i$ Estudis Avançats. This work was supported by grants from the Generalitat de Catalunya (2014 SGR 535) and the Spanish Ministerio de Ciencia e Innovación (MICINN) (SAF2013-46196) to RR Gomis. The authors have no other relevant affliations or financial involvement with any organization or entity with a financial interest in or financial conflict with the subject matter or materials discussed in the manuscript apart from those disclosed.

No writing assistance was utilized in the production of this manuscript.

\section{References}

1 Sotiriou C, Pusztai L. Gene-expression signatures in breast cancer. $N$. Engl J. Med. 360, 790-800 (2009).

2 Weigelt B, Peterse JL, van 't Veer LJ. Breast cancer metastasis: markers and models. Nat. Rev. Cancer 5, 591-602 (2005).

3 Nguyen DX, Bos PD, Massague J. Metastasis: from dissemination to organ-specific colonization. Nat. Rev. Cancer 9, 274-284 (2009).
4 Psaila B, Lyden D. The metastatic niche: adapting the foreign soil. Nat. Rev. Cancer 9, 285-293 (2009).

5 Paget $S$. The distribuition of secondary growths in cancer of the breast. Lancet 1, 571-573 (1889).

6 Nguyen DX, Massague J. Genetic determinants of cancer metastasis. Nat. Rev. Genet. 8, 341-352 (2007).

7 Minn AJ, Gupta GP, Siegel PM et al. Genes that mediate breast cancer metastasis to lung. Nature 436, 518-524 (2005).
8 Gupta GP, Nguyen DX, Chiang AC et al. Mediators of vascular remodelling co-opted for sequential steps in lung metastasis. Nature 446, 765-770 (2007).

9 Padua D, Zhang XH, Wang Q. TGFbeta primes breast tumors for lung metastasis seeding through angiopoietin-like 4. Cell 133, 66-77 (2008).

10 Chen Q, Zhang XH, Massague J. Macrophage binding to receptor VCAM-1 transmits survival signals in breast cancer 
cells that invade the lungs. Cancer Cell 20, 538-549 (2011).

11 Morales M, Arenas EJ, Urosevic J et al. RARRES3 suppresses breast cancer lung metastasis by regulating adhesion and differentiation. EMBO Mol. Med. 6, 865-881 (2014).

12 Kennecke H, Yerushalmi R, Woods R et al. Metastatic behavior of breast cancer subtypes. J. Clin. Oncol. 28, 3271-3277 (2010).

13 Myoui A, Nishimura R, Williams PJ et al. 2003. C-SRC tyrosine kinase activity is associated with tumor colonization in bone and lung in an animal model of human breast cancer metastasis. Cancer Res. 63, 5028-5033 (2003).

14 Smith MC, Luker KE, Garbow JR et al. CXCR4 regulates growth of both primary and metastatic breast cancer. Cancer Res. 64, 8604-8612 (2004).

15 Minn AJ, Kang Y, Serganova I et al. Distinct organ-specific metastatic potential of individual breast cancer cells and primary tumors. J. Clin. Invest. 115, 44-55 (2005).

16 Guise TA, Mohammad KS, Clines G et al. Basic mechanisms responsible for osteolytic and osteoblastic bone metastases. Clin. Cancer Res. 12, S6213-S6216 (2006).

17 Weilbaecher KN, Guise TA, McCauley LK. Cancer to bone: a fatal attraction. Nat. Rev. Cancer 11, 411-425 (2011).

18 Diel IJ. Prognostic factors for skeletal relapse in breast cancer. Cancer Treat. Rev. 27, 153-157; discussion 159-164 (2001).

19 Takagaki K, Takashima T, Onoda N et al. Parathyroid hormone-related protein expression, in combination with nodal status, predicts bone metastasis and prognosis of breast cancer patients. Exp. Ther. Med. 3 , 963-968 (2012).

20 Henderson MA, Danks JA, Slavin JL et al. Parathyroid hormone-related protein localization in breast cancers predict improved prognosis. Cancer Res. 66, 2250-2256 (2006)

21 Coleman RE. Bone Cancer in 2011: prevention and treatment of bone metastases. Nat. Rev. Clin. Oncol. 9, 76-78 (2011).

22 Pavlovic M, Arnal-Estape A, Rojo F et al. 2015. Enhanced MAF oncogene expression and breast cancer bone metastasis. J. Natl Cancer Inst. 107(12), djv256 (2015).
23 Mundy GR. Metastasis to bone: causes, consequences and therapeutic opportunities. Nat. Rev. Cancer 2, 584-593 (2002).

24 Tabaries S, Dupuy F, Dong Z et al. Claudin-2 promotes breast cancer liver metastasis by facilitating tumor cell interactions with hepatocytes. Mol. Cell Biol. 32, 2979-2991 (2012).

25 Kimbung S, Kovacs A, Bendahl PO et al. Claudin-2 is an independent negative prognostic factor in breast cancer and specifically predicts early liver recurrences. Mol. Oncol. 8, 119-128 (2014).

26 Bos PD, Zhang XH, Nadal C et al. Genes that mediate breast cancer metastasis to the brain. Nature 459, 1005-1009 (2009).

27 Valiente M, Obenauf AC, Jin X et al. Serpins promote cancer cell survival and vascular co-option in brain metastasis. Cell 156, 1002-1016 (2014).

28 Baselga J, Bradbury I, Eidtmann $\mathrm{H}$ et al. Lapatinib with trastuzumab for HER2positive early breast cancer (NeoALTTO): a randomised, open-label, multicentre, Phase 3 trial. Lancet 379, 633-640 (2012). 